\title{
Gamma-ray shielding effectiveness of lead bismuth germanoborate glasses
}

\begin{abstract}
The gamma-ray shielding effectiveness of the lead bismuth germanoborate glasses has been studied. The mass attenuation coefficients $(\mu / \rho)$ of the selected glasses have been obtained through both XCOM program and MCNP5 simulation code. The $(\mu / \rho)$ values calculated in both methods are found to be in good agreement and these values are used to calculate effective atomic number, mean free path, half-value layer and energy exposure buildup factors. The shielding effectiveness of these samples has been compared with that of window glasses and some standard shielding concretes. The lower values of mean free path point to the fact that the selected glasses are efficient gamma shields.
\end{abstract}

Keyword: Glass; Mass attenuation coefficient; Radiation shielding; XCOM; MCNP5 code 
